\section{Against a role for attentional disengagement in the gap effect: A friendly amendment to Tam and Stelmach (1993)}

\author{
RAYMOND M. KLEIN and TRACY L. TAYLOR \\ Dalhousie University, Halifax, Nova Scotia, Canada \\ and \\ ALAN KINGSTONE \\ University of Alberta, Edmonton, Alberta, Canada
}

Saccadic reaction time ( $R T)$ is reduced when the fixation point is removed shortly before target onset. Although Tam and Stelmach (1993) argued that this gap effect could not be explained solely by the idea that fixation offset disengaged visual attention and preferred an explanation based on disengagement of the oculomotor system, they felt that they could not mule out a hybrid model in which both oculomotor and attentional disengagement contribute to the gap effect. Our analysis of the dual response experiment (Experiment 4), upon which this hybrid model was based, shows that manual and saccadic responses were likely compromised by a grouping or delay strategy and that subjects may not have been attending as instructed. On these grounds, we argue that Tam and Stelmach (1993), like Kingstone and Klein (1990; 1993a) provide no evidence that attentional disengagement contributes to the gap effect. An alternative proposal (Klein \& Kingstone, 1993), that motor preparation and oculomotor disengagement combine additively to produce the gap effect, is consistent with the data from Tam and Stelmach's Experiments 1-3, is similar to the explanation that they prefer, and has been strongly supported when $d i$ rectly tested (Kingstone, Klein, \& Taylor, 1994).

Saccadic latencies are reduced when fixation offset precedes target onset (Fendrich, Hughes, \& ReuterLorenz, 1991; Fischer, 1987; Fischer \& Boch, 1983; Fischer \& Ramsperger, 1984; Hallett \& Adams, 1980; Kingstone \& Klein, 1993b; Mayfrank, Mobashery, Kimmig, \& Fischer, 1986; Reulen, 1984; Reuter-Lorenz, Hughes, \& Fendrich, 1991; L. E. Ross \& S. M. Ross, 1980; S. M. Ross \& L. E. Ross, 1981; Saslow, 1967; Wenban-Smith \& Findlay, 1991). Foremost among explanations of this "gap effect" are those which attribute the reduction in reaction time to the prior disengagement of covert attention that is afforded by the offset of the fixation stimulus (e.g., Braun \& Breitmeyer, 1988; Fischer, 1987; Fischer \& Breitmeyer, 1987; Mayfrank et al., 1986) and those which attribute the reduction in reaction

This research was supported by a Natural Sciences and Engineering Research Council of Canada (NSERCC) Fellowship Award to A.K. and an NSERCC Operating Grant to R.M.K. Correspondence concerning this article should be addressed to R. M. Klein, Department of Psychology, Dalhousie University, Halifax, Nova Scotia, Canada B3H 4J1 (e-mail: klein@ac.dal.ca). time to the disengagement of the oculomotor system that is likely mediated by activity in the superior colliculus (e.g., Klein, 1993; Taylor, Kingstone, \& Klein, 1993). Tam and Stelmach (1993; hereafter, T\&S) recently reported five experiments that were intended to examine the contributions of covert attentional and oculomotor disengagement to the gap effect. They interpreted their results as contrary to a purely covert attention explanation of the gap effect, but suggested that the findings of their Experiment 4 were consistent with either an ocular or a hybrid attention-ocular explanation. Citing parsimony, T\&S preferred the ocular disengagement explanationa choice with which we concur, but for different reasons.

In the present commentary we review the T\&S experiments, giving special emphasis to the critical Experiment 4 , in which the locus of attention appeared to influence the magnitude of the gap effect. We argue that the assessment of attentional allocation was compromised in that experiment, making it a weak basis for statements about attentional influence. Moreover, Kingstone and Klein $(1990,1993 a)$ found no influence of the direction of covert attention on the gap effect. Thus, there is no convincing evidence to support a role for attentional disengagement in the gap effect. A model that treats the gap effect as composed of two independent components - oculomotor disengagement and general response preparation - can account for our previous findings and those of $\mathrm{T} \& \mathrm{~S}$ (Experiments 1-3).

\section{Tam and Stelmach (1993)}

The basic findings of T\&S are reported in their Experiments 1-3. While the subject was under instruction to attend covertly to a single peripheral stimulus positioned above or below a central fixation stimulus, offset of the fixation stimulus produced a greater reduction in saccadic latencies than did offset of the peripheral stimulus (Experiments 1 and 3 ). In contrast, these fixation and peripheral offsets had equivalent facilitatory effects on manual responses (Experiments 2 and 3). Because the saccadic findings are inconsistent with the attentional disengagement prediction that an attended peripheral offset will decrease saccadic latencies whereas an unattended fixation offset will not, T\&S rejected a purely attentional disengagement explanation of the gap effect (see Kingstone \& Klein, 1990, 1993a; Klein, Kingstone, \& Pontefract, 1992, for a similar conclusion).

A shortcoming of these first three experiments was that an objective measurement of covert orienting was never taken. Experiment 4 incorporated a dual-task paradigm to address this problem, with a saccadic and a manual response required on most trials. Subjects were instructed to fixate a central stimulus and to direct their attention covertly at fixation or to a single peripheral stimulus that was positioned above or below the fixation stimulus. This attention manipulation-fixation versus 
periphery - was constant for a block of trials. Subjects were instructed to press a button as quickly as possible when the temporally unpredictable offset of a peripheral or fixation stimulus was detected, and to execute a saccade as quickly as possible to the onset of a stimulus that appeared randomly to the left or right of center. Overlap trials, in which an offset event did not occur, served as catch trials for the manual task.

The rationale of T\&S is sound: "We would expect that in sessions in which the subjects were instructed to attend to the foveal stimulus, manual latencies would be shorter to the offset of the foveal stimulus than to the offset of the eccentric stimulus. Similarly, in sessions in which the subjects were instructed to attend to the eccentric stimulus, manual latencies should be shorter to the offset of the eccentric stimulus" (p. 216). Their results are presented in Figure 1.

The manual reaction time (RT) data were as predicted. When subjects were instructed to attend peripherally, RTs were approximately $100 \mathrm{msec}$ faster to a peripheral offset than to a fixation offset; in the attend fixation condition, RTs were approximately $50 \mathrm{msec}$ faster to a fixation offset than to a peripheral offset. These data were interpreted as indicating that attention was oriented in line with instruction.

The effect of visual offsets on saccadic performance was measured against the overlap (no offset event) baseline condition. In the attend peripheral condition, saccadic latencies were reduced when an offset (manual target) event coincided with the onset of a saccadic target ( 0 -msec gap condition), and this facilitation grew as the interval between an offset event and the onset of a target increased $(100,150,200,300 \mathrm{msec})$. This data pattern occurred for both peripheral and fixation offsets. In the attend fixation condition, the same data pattern was also observed, with the exception that there was no RT reduction at the 0 -msec peripheral offset gap condition (i.e., when peripheral offset and target onset coincided, saccadic RT was the same as when there was no offset event at all). Thus, with this one exception, all offset events facilitated saccadic RT. Moreover, as in Experiments 1-3, fixation offset produced a greater reduction in saccadic RT than a peripheral offset, regardless of where subjects were asked to attend. However, attentional instruction did modulate the size of the fixation offset and peripheral offset effects: When measured

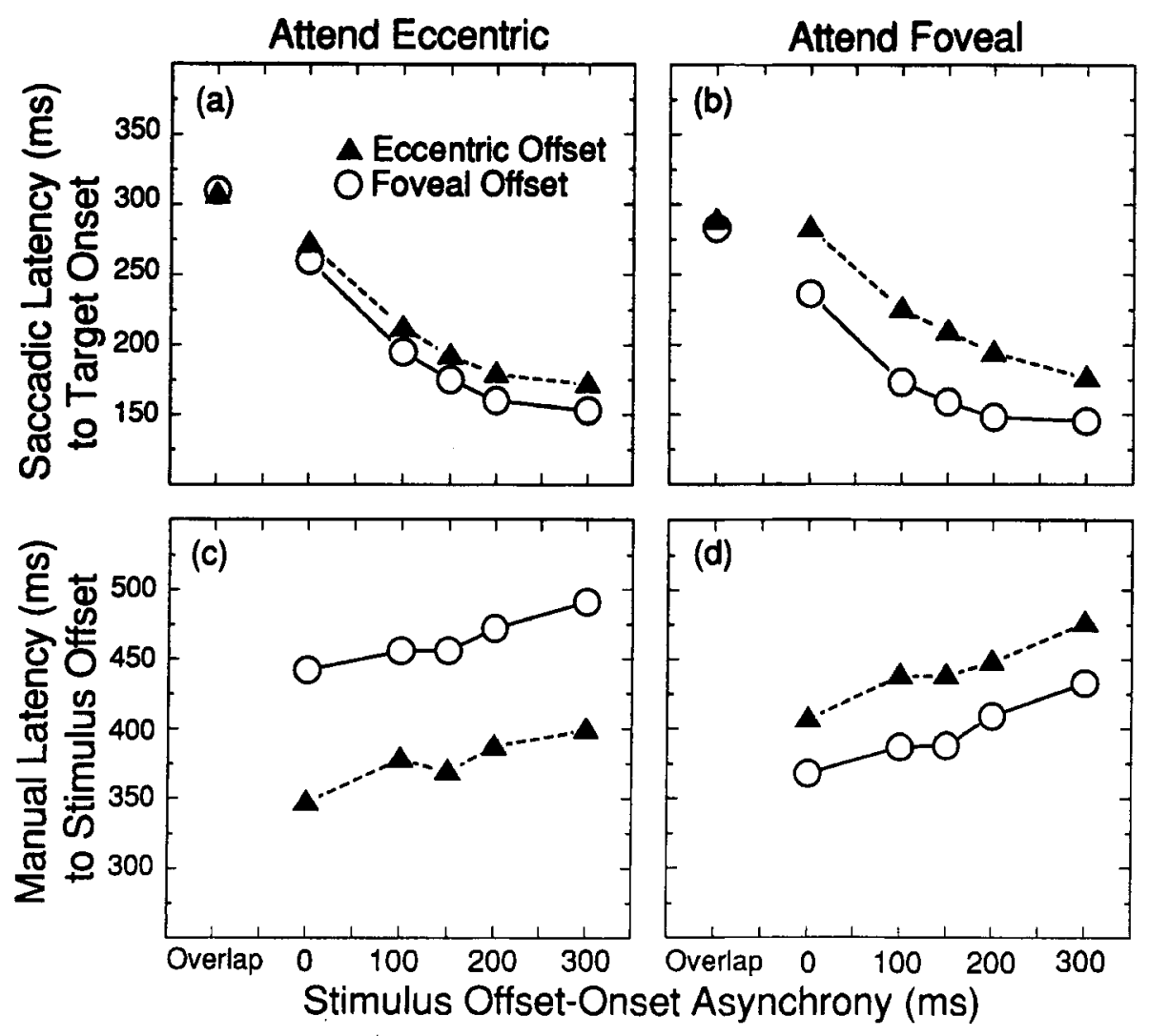

Figure 1. (a, b) Saccadic RTs to target onset and (c, d) manual RTs to stimulus offset as a function of locus of presumed attentional locus and stimulus offset-onset asynchrony (in milliseconds). From "Vlewing Behavior: Ocular and Attentional Disengagement," by W. J. Tam \& L. B. Stelmach, 1993, Perception \& Psychophysics, 54, p. 217. Copyright 1993 by Psychonomic Society, Inc. Reprinted by permission. 
against the overlap baseline, fixation offset facilitated saccadic latencies more in the attend fixation than in the attend peripheral condition, and conversely, peripheral offset facilitated saccadic latencies more in the attend peripheral than in the attend fixation condition.

According to the ocular explanation, as formulated by $\mathrm{T} \& \mathrm{~S}$, the eye movement system is engaged when a fixation stimulus is foveated. A saccade to a peripheral stimulus requires that the ocular system first be disengaged from fixation. Fixation offset disengages the ocular system, thereby reducing saccadic RT when fixation offset precedes (or even coincides with) target onset. A peripheral offset will also reduce saccadic latency, but to a lesser degree, because ocular disengagement takes longer when a foveal stimulus is still present. The ocular explanation fails, however, to explain why attentional instruction modulated the size of the fixation offset and peripheral offset gap effects in Experiment 4. To incorporate this result within the ocular disengagement framework, T\&S suggested that an offset might have been detected sooner at the attended location than at the unattended location. Implicit in this explanation is the idea that part of the gap effect may reflect a warning signal/response preparation effect that begins following detection of the offset. T\&S recognized this possibility (they called it alerting) but were not explicit about the role that this factor played in the gap effect.

The results of Experiment 4 also suggested to T\&S an alternative explanation that combined ocular and attentional processes. This explanation is based on three assumptions: (1) that both the ocular and the covert attention systems must disengage before an eye movement can be executed; (2) that disengagement of the two systems occurs in parallel; and (3) that oculomotor disengagement is slower than attentional disengagement. Although T\&S favored the more parsimonious ocular explanation, they were unable to distinguish between it and the ocular attention account.

\section{An Analysis of Experiment 4}

Because manual responses were faster to attended than to unattended offsets, T\&S concluded that subjects were attending as instructed. Saccadic latency differences in the attend fixation and attend peripheral conditions might therefore reflect the influence of attentional disengagement. However, a close examination of Experiment 4 leads us to question this conclusion. Of particular concern is the fact that there was an increase in manual RTs (50-msec attend peripheral, $75-\mathrm{msec}$ attend fixation) with increasing gap duration. In other words, the longer the delay between an offset event (the manual target) and the onset of a subsequent saccadic target, the longer it took subjects to make the manual response to the offset event. This result implies that on a proportion of trials subjects were withholding the manual response until a saccadic target appeared, perhaps sometimes grouping the initiation of manual and saccadic responses (Kantowitz, 1974, pp. 117-119)—a possibility that is supported by the fact that saccadic responses were executed before manual responses at all but the longest gap durations (manual responses occurred as much as $150 \mathrm{msec}$ after saccadic responses). Our position is that the delay in manual RT relative to saccadic RT, and the response strategy that it must reflect, undermines reliance on manual RT for assessing the direction of attention at the time of an offset event. In other words, while T\&S attribute the effect of attentional instruction upon manual RT to covert orienting to the peripheral stimulus, they cannot rule out the possibility that subjects were delaying the manual responses according to the attentional instruction.

There is also a possible methodological problem with Experiment 4: Subjects in that experiment may not have been consistently fixating the appropriate stimulus when attending to the peripheral stimulus. Subjects initiated each trial "when they felt confident that they were attending properly" (p. 217). T\&S's (1993) eye position monitoring procedure was designed to detect saccades, but it could not ensure that the subjects were fixating where instructed (Stelmach, personal communication). During free inspection of a scene, subjects have relatively poor introspective awareness of where they are looking (see, e.g., Kaufman \& Richards, 1969). It is therefore possible that on some proportion of the trials in the attend eccentric condition, subjects fixated the eccentric stimulus instead of the fixation stimulus. 'If this were the case, there would be no need to postulate a role for attentional disengagement in the gap effect to explain the data from this experiment: On the attend peripheral trials during which subjects fixated the peripheral stimulus, offset of the (unfixated) central stimulus would not disengage the oculomotor system, whereas offset of the (fixated) peripheral stimulus would. This would have the observed effect of reducing saccadic latencies on some of the peripheral offset trials and increasing the latencies on some of the fixation offset trials in the attend peripheral condition.

Finally, Kingstone and Klein $(1990,1993 a)$, in a series of experiments that are not vulnerable to the problems just outlined, tested and rejected the hypothesis that the gap effect is influenced by the locus of attention. They presented subjects with cues (endogenous or exogenous) to one of two peripheral locations (above and below fixation) at which a target demanding a speeded manual detection response could occur on half of all trials. On the remaining trials, a manual target was not presented and subjects were required to saccade to the right or left of fixation, in response to an onset target that appeared to the right or left of fixation. Having thus encouraged subjects to attend to one of the two peripheral stimuli (and having confirmed attentional allocation through cost-benefit analysis), Kingstone \& Klein (1990, 1993a) could determine the effects of offsetting (1) the fixation stimulus, (2) the attended peripheral stimulus; and (3) the unattended peripheral stimulus. Cost-benefit analysis of manual RT confirmed that subjects attended 
to the cued peripheral stimulus, and since fixation was achieved before the attentional instruction was given, any trials with gaze shifts toward the cued location would be detected. Unlike T\&S's (1993) results, Kingstone and Klein's $(1990,1993 a)$ revealed no latency differences between saccades that followed offset of the attended peripheral stimulus and those that followed offset of the unattended peripheral stimulus.

To summarize, T\&S's subjects' manual responses in Experiment 4 were not executed solely (if at all) to the offset event, and therefore their manual RTs cannot provide a reliable index of the locus of attention at the time of the offset. Moreover, whatever gap-duration-sensitive strategy produced this delay (e.g., response grouping) may have contaminated the saccadic data. Finally, it is possible that subjects were sometimes fixating what was supposed to have been an attended eccentric stimulus. These weaknesses, together with Kingstone and Klein's $(1990,1993 a)$ repeated finding that the gap effect is uninfluenced by the direction of attention, suggest no role for attentional disengagement in the gap effect (see Inhoff, Topolski, Vitu, \& O'Regan, 1993, for converging evidence).

\section{Two-Component Model of the Gap Effect}

Why are saccadic latencies reduced when fixation offset precedes target onset? We have proposed that two components combine additively to produce this gap effect (Kingstone \& Klein, 1993a; Klein \& Kingstone, 1993; see Kingstone, Klein, \& Taylor, 1994, for a discussion of similar ideas put forward by Reuter-Lorenz et al., 1991, and by L. E. Ross \& S. M. Ross, 1980; S. M. Ross \& L. E. Ross, 1981). One component, which follows any offset event, is motor preparation. This can operate on any response modality and is primarily dependent on the foreperiod between a warning signal and a subsequent target (in our experiments, this component varies from 0 to $60 \mathrm{msec}$, depending on the subject's level of alertness and the response information conveyed by offsets). The other component, which is specific to fixation offsets, reduces saccadic latencies by freeing the oculomotor system from fixation (in our experiments, this component is relatively stable at about $35 \mathrm{msec}$ ). This component, which we call the fixation offset effect, is probably mediated by inhibition from the rostral pole of the superior colliculus (Munoz \& Wurtz, 1993). This two-component model predicts that: (1) the latency of any response may be reduced by an offset event, with the degree of reduction being dependent on the foreperiod duration, type of response, and response information conveyed by the offset; (2) only saccadic responses will enjoy the additional benefit from fixation offsets (fixation offset effect); and because they operate at different stages of processing, (3) the two effects will combine additively when the offset event is at fixation. Our own results have borne out all of these predictions (Taylor et al., 1993; see also Kingstone et al., 1994), as does the pattern of saccadic and manual response latencies in T\&S's (1993) Experiments 1-3.

\section{REFERENCES}

Braun, D., \& Breitmeyer, B. G. (1988). Relationship between directed visual attention and saccadic reaction times. Experimental Brain Research, 73, 546-552.

Fendrich, R., Hughes, H. C., \& Reuter-LoRenz, P. A. (1991). Fixationpoint offsets reduce the latency of saccades to acoustic targets. Perception \& Psychophysics, 50, 383-387.

FISCHER, B. (1987). The preparation of visually guided saccades. Review of Physiology, Biochemistry, \& Pharmacology, 106, 1-35.

Fischer, B., \& Boch, R. (1983). Saccadic eye movements after extremely short reaction times in the monkey. Brain Research, 260 , 21-26.

FISCHER, B., \& BREITMEYER, B. (1987). Mechanisms of visual attention revealed by saccadic eye movements. Neuropsychologia, 25, 73-83.

FisCHER, B., \& RAMSPERGER, E. (1984). Human express saccades: Extremely short reaction times of goal directed eye movements. $E x$ perimental Brain Research, 57, 191-195.

HALLETT, P. E., \& ADAMs, B. D. (1980). The predictability of saccadic latency in a novel voluntary oculomotor task. Vision Research, 20, 329-339.

Inhoff, A. W., Topolski, R., Vitu, F., \& O'Regan, J. K. (1993). Attention demands during reading and the occurrence of brief (express) fixations. Perception \& Psychophysics, 54, 814-823.

KanTowitz, B. (1974). Double stimulation. In B. H. Kantowitz (Ed.), Human information processing: Tutorials in performance and cognition (pp. 83-132). Hillsdale, NJ: Erlbaum.

Kaufman, L., \& Richards, W. (1969). Spontaneous fixation tendencies for visual forms. Perception \& Psychophysics, 5, 85-88.

Kingstone, A., \& Klein, R.M. (1990, November). Attention and express saccades. Paper presented at the meeting of the Psychonomic Society, New Orleans.

KingSTONE, A., \& KLEIN, R. M. (1993a). Visual offsets facilitate saccadic latency: Does predisengagement of visuospatial attention mediate this gap effect? Journal of Experimental Psychology: Human Perception \& Performance, 19, 1251-1265.

Kingstone, A., \& KLEIN, R. M. (1993b). What are human express saccades? Perception \& Psychophysics, 54, 260-273.

Kingstone, A., KLEIN, R. M., \& TAYLOR, T. L. (1994). Visual offsets facilitate saccadic latency: There are two components to this gap effect. Unpublished manuscript.

KLEIN, R. M. (1993, May). On the relationships between overt and covert orienting: A view from human performance. Paper presented at the West Coast Attention Meeting, Eugene, OR.

KLEIN, R. M., \& KIngSTONE, A. (1993). Why do visual offsets reduce saccadic latencies? Behavioral \& Brain Sciences, 16, 583-584.

Klein, R. M., Kingstone, A., \& Pontefract, A. (1992). Orienting of visual attention. In K. Rayner (Ed.), Eye movements and visual cognition (pp. 46-65). New York: Springer-Verlag.

Mayfrank, L., Mobashery, M., Kimmig, H., \& Fischer, B. (1986). The role of fixation and visual attention in the occurrence of express saccades in man. European Archives of Psychiatry \& Neurological Science, 235, 269-275.

MunOz, D. P., \& WuRTz, R. H. (1993). Fixation cells in monkey superior colliculus: II. Reversible activation and deactivation. Journal of Neurophysiology, 70, 576-589.

REULEN, P. H. (1984). Latency of visually evoked saccadic eye movements: I. Saccadic latency and the facilitation model. Biological Cybernetics, 50, 251-262.

Reuter-Lorenz, P. A., Hughes, H. C., \& Fendrich, R. (1991). The reduction of saccadic latency by prior offset of the fixation point: An analysis of the gap effect. Perception \& Psychophysics, 49, 167175.

Ross, L. E., \& Ross, S. M. (1980). Saccade latency and warning signals: Stimulus onset, offset, and change as warning events. Perception \& Psychophysics, 27, 251-257. 
Ross, S. M., \& Ross, L. E. (1981). Saccadic latency and warning signals: Effects of auditory and visual stimulus onset and offset. Perception \& Psychophysics, 29, 429-437.

SAsLow, M. G. (1967). Effects of components of displacement-step stimuli upon latency for saccadic eye movement. Journal of the Optical Society of America, 57, 1024-1029.

TAM, W. J., \& STElmaCH, L. B. (1993). Viewing behavior: Ocular and attentional disengagement. Perception \& Psychophysics, 54, 211 222.

TAylor, T., Kingstone, A. F., \& Klein, R. M. (1993, July). Visual offsets facilitate saccadic latency: There are two components to this gap effect. Paper presented at the meeting of the Canadian Society for Brain, Behavior and Cognitive Science, Toronto.
Wenban-SMith, M. G., \& Findlay, J. M. (1991). Express saccades: Is there a separate population in humans? Experimental Brain Research, 87, 218-222.

\section{NOTE}

1. Although subjects are able to fixate accurately, it has not been shown that this ability generalizes to situations in which fixation and attention instructions conflict.

(Manuscript received February 1, 1994;

revision accepted for publication October 31, 1994.) 